\title{
Analysis of Three-phase Short Circuit Current by Three Elements Method
}

\author{
Ye Tang* \\ North China Electric Power University, Changping District, Beijing \\ ${ }^{*}$ Corresponding author
}

\begin{abstract}
In this paper, we use the knowledge of the three elements method to analyze the steady-state and transient conditions of three-phase short-circuit current. The method combine actual power system conditions with the circuit subject knowledge. Compared with the traditional solution of three-phase short circuit current, it avoid solving first-order linear inhomogeneous differential equation, which is a new idea to analyze the problem of three-phase short circuit.
\end{abstract}

Keywords-three-phase; short circuit; three elements method; steady-state conditions; transient conditions

\section{INTRODUCTION}

Short circuit fault is a common fault in the power system. It changes the circuit structure, causing changes in power distribution, which will bring energy loss, damage to the stability of the power system, affecting the normal operation of electrical equipment. Three-phase short circuit is one of the most common short-circuit fault. It is of great significance to study the change of current in three-phase sudden short circuit to the power system.

\section{Assumptions}

We assume that the power of the power is infinite, the power change caused by the external circuit is negligible, and so the voltage and frequency remain constant. Infinitely large power can be seen as a combination of multiple finite power supplies in parallel, so the internal impedance is zero. When the internal impedance of the power supply is less than $10 \%$ of the total impedance of the short circuit, it can be approximately considered that the power supply is an infinitely high power supply.

\section{SHORT CIRCUIT ANALYSIS}

Since the three-phase short circuit is symmetrical, we only need to analyze one phase circuit. Here we take a phase as the research object.

According to the three factor method, the full response of the circuit consists of steady-state response and transient response.

$$
f(t)=f_{\infty}(t)+\left(f(0)-f_{\infty}(0)\right) \times e^{-\frac{t}{T}}
$$

The three factor method is applied to the three-phase short circuit, we can see, three-phase short-circuit current also by the periodic components and non-periodic components.

$$
\mathrm{i}_{a}(t)=i_{a \infty}(t)+\left(i_{a}(0)-i_{a \infty}(\infty)\right) \times e^{-\frac{t}{T}}
$$

The periodic component is the case when the three-phase short-circuit current is in steady state and can be called the steady-state short-circuit current.

$$
\mathrm{i}_{a \infty}(t)=\frac{U}{Z} \times \sin (\omega t+\alpha-\varphi)
$$

$\varphi$ is the impedance angle of the entire line after the short circuit, $\alpha$ is the initial phase angle of the power supply. Then we analyze the transient process.

$$
\mathrm{i}_{\mathrm{a}}(0)=I_{m(0)} \sin \left(\omega t+\alpha-\varphi_{0}\right)
$$

$\varphi_{0}$ is the power factor angle before short-circuiting, $\alpha$ is the initial phase angle of the power supply.

$$
\mathrm{i}_{a \infty}(0)=\frac{U}{Z} \times \sin \left(\alpha-\varphi_{0}\right)=I_{m} \sin \left(\alpha-\varphi_{0}\right)
$$

Bring (3), (4) and (5) into equation (2),we get the final expression for the three-phase short circuit current

$$
\begin{aligned}
& \mathrm{i}_{a}=I_{m} \sin (\omega t+\alpha-\varphi)+ \\
& \left(I_{m 0} \sin \left(\alpha-\varphi_{0}\right)-I_{\mathrm{m}} \sin (\alpha-\varphi)\right) \times e^{-\frac{t}{T}}
\end{aligned}
$$

Compared with the analytical methods commonly used in power system analysis, the three-element method is more concise and intuitive, avoiding the calculation of differential and integral.

\section{SUMMARY}

In this paper, we use the knowledge of the three elements of the equivalent circuit method to analyze the three-phase short-circuit current steady-state and transient conditions. The power system conditions can be combined with the circuit subject knowledge to avoid solving first-order linear inhomogeneous differential equation, which is a new idea to analyze the problem of three-phase short circuit. 


\section{$\mathrm{V}$ ACKNOWLEDGEMENT}

At last, I'd like to express my sincere thanks to all those who have lent me hands in the process of my writing this paper. In particular, I would like to show my deepest gratitude to my colleague, Xuan WU, who has provided me with valuable guidance in every stage of the writing of this paper without his help, I could not have completed my thesis.

\section{REFERENCES}

[1] GuanYuan Qiu, Circuit [M]. Higher Education Press, China, 1989.

[2] G. R. Li, Basis of Power System Analysis, first ed., CHINA MACHINE PRESS, China, 2015.pp. 172-173.

[3] HongLing Lei, QingHua Li .Analysis of Three-phase Short Circuit in Infinite Capacity Power Supply System. Journal of Maoming College, (2002).

[4] Xi-jun CHEN. Discussion on the Calculation of Three-phase Short-circuit Current .Journal of Huanggang Polytechnic, (2012)

[5] Information on https://www.ieee.org/index.html. 